\title{
Dermatitis herpetiformis: pathophysiology, clinical presentation, diagnosis and treatment ${ }^{*}$
}

\author{
Marcos Vinícius Clarindo ${ }^{1}$ \\ Emylle Marlene Soligo ${ }^{1}$ \\ Roseli Terezinha Ruaro ${ }^{1}$
}

\author{
Adriana Tomazzoni Possebon ${ }^{1}$ \\ Hirofumi Uyeda ${ }^{1}$ \\ Julio Cesar Empinotti ${ }^{1}$
}

DOI: http://dx.doi.org/10.1590/abd1806-4841.20142966

\begin{abstract}
Researches on DH have shown that it is not just a bullous skin disease, but a cutaneous-intestinal disorder caused by hypersensitivity to gluten. Exposure to gluten is the starting point of an inflammatory cascade capable of forming autoantibodies that are brought to the skin, where they are deposited, culminating in the formation of skin lesions. These lesions are vesico-bullous, pruritic, and localized especially on elbows, knees and buttocks, although atypical presentations can occur. Immunofluorescence of perilesional area is considered the gold standard for diagnosis, but serological tests help in cases where it is negative. Patients who follow glutenfree diets have better control of symptoms on the skin and intestine, as well as lower risks of progression to lymphoma. Dapsone remains the main drug for treatment, but it requires monitoring of possible side effects, some potentially lethal.
\end{abstract}

Keywords: Celiac disease; Dapsone; Dermatitis herpetiformis; Diet, gluten-free; Transglutaminases

\section{INTRODUCTION}

Dermatitis Herpetiformis (DH), also known as Duhring-Brocq dermatitis, is a chronic, recurrent disease, secondary to gluten hypersensitivity which main clinical manifestation is the occurrence of a papulovesicular pruriginous rash. ${ }^{1}$ Celiac Disease (CD) is the gastrointestinal manifestation of the same etiology and it is characterized by atrophy of the intestinal mucosa secondary to gluten-containing diet. DH patients rarely have gastrointestinal symptoms, but they generally present some degree of intestinal villous atrophy. ${ }^{2}$

$\mathrm{DH}$ is characterized by the presence of $\operatorname{IgA}$ deposits on top of the dermal papillae and manifests itself mainly on the extensor surface of the limbs, buttocks and scapular area. ${ }^{3}$ High incidence of autoimmune diseases and potential complications, including the development of lymphoma have been demonstrated in this dermatosis. ${ }^{4}$

Patients with $\mathrm{DH}$ and $\mathrm{CD}$ may have the same association with histocompatibility antigens (HLA), the presence of circulating IgA against transglutaminase autoantigens and clinical remission on gluten-free diet. ${ }^{5}$

\section{HISTORY}

Dermatologist Louis A. Duhring first described this dermatosis in 1884. It is likely that some cases initially described were of bullous pemphigoid or linear IgA dermatosis bullosa, given the unavailability of better diagnostic tools at that period. ${ }^{6}$

Costello first described, in 1940, the effectiveness of sulfapyridine in a patient with this dermatosis. In 1943 Civatte demonstrated that, in pemphigus, the formation of blisters was intraepidermal, differently from bullous pemphigoid and $\mathrm{DH}$, which present subepidermal alterations. ${ }^{6}$

In the 1950s Pierard described the presence of clusters of neutrophils and eosinophils in the dermal papillae, especially in more recent lesions. In 1969 Van der Meer, in turn, revealed the occurrence of granular IgA deposits in those same locations, which constituted a major milestone for the understanding of this disease and permitted for the first time its differentiation from linear IgA dermatosis bullosa.?

Fry described the association with gluten hypersensitivity in 1967, whereas clinical improvement of cutaneous manifestations with gluten deprivation was noted in $1973 .{ }^{3,8,9}$

Received on 13.07.2013

Approved by the Advisory Board and accepted for publication on 07.08.2013

* Work performed at West Paraná State University (UNIOESTE) - Cascavel (PR), Brazil.

Financial Support: None.

Conflict of Interest: None.

West Paraná State University (UNIOESTE) - Cascavel (PR), Brazil.

(C2014 by Anais Brasileiros de Dermatologia 
In 1978, Strober and Katz elucidated the association between HLA B8/DR3 with CD and DH, and in 1987 Kumar et al first noticed the presence of antiendomysial antibodies in both diseases. ${ }^{10}$

\section{EPIDEMIOLOGY}

A relatively rare disease; it is more prevalent in Scandinavian countries and in the UK. Studies conducted in Scotland and Sweden found an incidence of 11.5 and 19.6 affected individuals per 100,000 inhabitants, respectively. ${ }^{11,12}$ The higher incidence ever recorded was in Ireland, 1 person for every 300 inhabitants. It affects predominantly Caucasians compared to African-Americans or Asians. In the latter, besides being rare, the disease is characterized by often not being associated with $\mathrm{CD}$, besides the prevalence of fibrillar IgA deposits on direct immunofluorescence, and a different HLA pattern. ${ }^{3,5,6}$

DH may occur at all ages, but most cases affect young adults, between 15 and 40 years old. On gender incidence, males predominate in a ratio of 3:2 compared to females, but in younger individuals this ratio is reversed, with affected females being more prevalent.

\section{PATHOGENESIS}

\section{Genetic factors:}

Studies in monozygotic twins suggest a common genetic basis between DH and CD. Hervonen evaluated 6 pairs of twins, and noted that 3 pairs had $\mathrm{DH}$, and in 2 pairs one twin had $\mathrm{DH}$ and the other had $\mathrm{CD}$, and also in just a pair, one twin had $\mathrm{DH}$ and the other none of the diseases. Despite the probable similar genetic origin, environmental factors may influence the occurrence of either pathology. ${ }^{2}$ Approximately $5 \%$ of patients with $\mathrm{DH}$ have a sibling with the same pathology, and the percentages for CD are even higher. ${ }^{7,13}$

In both $\mathrm{CD}$ and $\mathrm{DH}$, HLA DQ2 or HLA DQ8 alleles are inherited, this being the likely genetic base for the association, which is also observed in animals. ${ }^{5}$ The presence of both alleles provide a sensitivity close to $100 \%$ with a high negative predictive value, i.e., individuals which do not carry any of the alleles have the diagnoses of $\mathrm{CD}$ and $\mathrm{DH}$ excluded. . $^{5,714}$

\section{Triggering factors:}

The major environmental factor involved in triggering the disease is exposure to gluten. $\mathrm{DH}$ and $\mathrm{CD}$ are significant examples of pathologies in which environmental factors participate in the physiopathogeny. ${ }^{5,15}$ Gluten is composed of two peptides, gliadin and glutenin, with the disease pathogenesis being linked to gliadin. It can be classified according to its electrophoretic mobility into 4 groups: $\alpha, \beta, \delta$ and $\lambda$. The fraction linked to intestinal disease is from the $\alpha$-gliadin group, and its immunoreactivity is due to the N-terminal. ${ }^{5}$ Topical or intradermal application of gluten is not sufficient to trigger typical DH lesions, demonstrating that the development of this disease involves intestinal exposure to gluten. ${ }^{3}$

\section{Immunological response:}

a) Transglutaminase family and IgA deposits:

In addition to antibodies directed precisely against gliadin in the intestinal mucosa, the formation of specific antibodies against autoantigens, such as transglutaminases, may also occur. Dieterich et al first described them in 1997, suggesting that this was the primary autoantigen recognized by IgA in $\mathrm{CD}$ and $\mathrm{DH} .{ }^{6,16,17}$

The transglutaminase family consists of nine different types of proteins expressed in various cell types. Two of them are relevant in DH: tissue transglutaminase (TTG) and epidermal transglutaminase (ETG). TTG is widely distributed in the human body, and is considered a surrogate marker for CD diagnosis. Many authors have demonstrated that TTG's enzymatic activity may be part of the pathogenesis of several diseases such as Huntington's disease, Alzheimer's disease and also CD. ${ }^{18,19,20}$

Sardy et al first described ETG in 2002. ${ }^{21}$ It is present in keratinocytes and among its functions we highlight the maintenance of stratum corneum's integrity. Also known as transglutaminase 3, it performs its function by connecting the various epidermal structural proteins. ${ }^{22}$ TTG is the main antigen for $\mathrm{CD}$ antibodies, as ETG is the antigen in DH. Anti-TTG antibodies may, by cross-reaction, recognize ETG, leading to the onset of cutaneous IgA deposits (Figure 1). Between TTG and ETG molecules there is $64 \%$ structural homology, which would explain the occurrence of cross-reaction. Serum from patients with $C D$ react against TTG and ETG, whilst those of patients with $\mathrm{DH}$ react mainly against ETG. ${ }^{21,23-26}$

In normal subjects ETG is found in more superficial epidermal keratinocytes, and not in the dermoepidermal junction, the main site of IgA deposits. ${ }^{21}$ There are two hypotheses to explain this phenomenon. After a trauma, keratinocytes might release ETG that would then deposit in the basement membrane. Circulating antibodies would bind to these autoantigens forming the disease's characteristic deposits in that site. ${ }^{27}$ A second hypothesis is the possibility that keratinocytes would release ETG into the blood stream, where it would form immune complexes with $\operatorname{Ig} \mathrm{A}$, which would then deposit in the dermal papillae. ${ }^{21}$ There are reports of IgA nephropathy in patients with $\mathrm{DH}$, reinforcing the latter possibility. ${ }^{28,29} \mathrm{~A}$ study by Donaldson et al showed that, in the absence of IgA, ETG is not found in the dermis, which favors the hypothesis of the deposit mechanism..$^{23,27}$ 


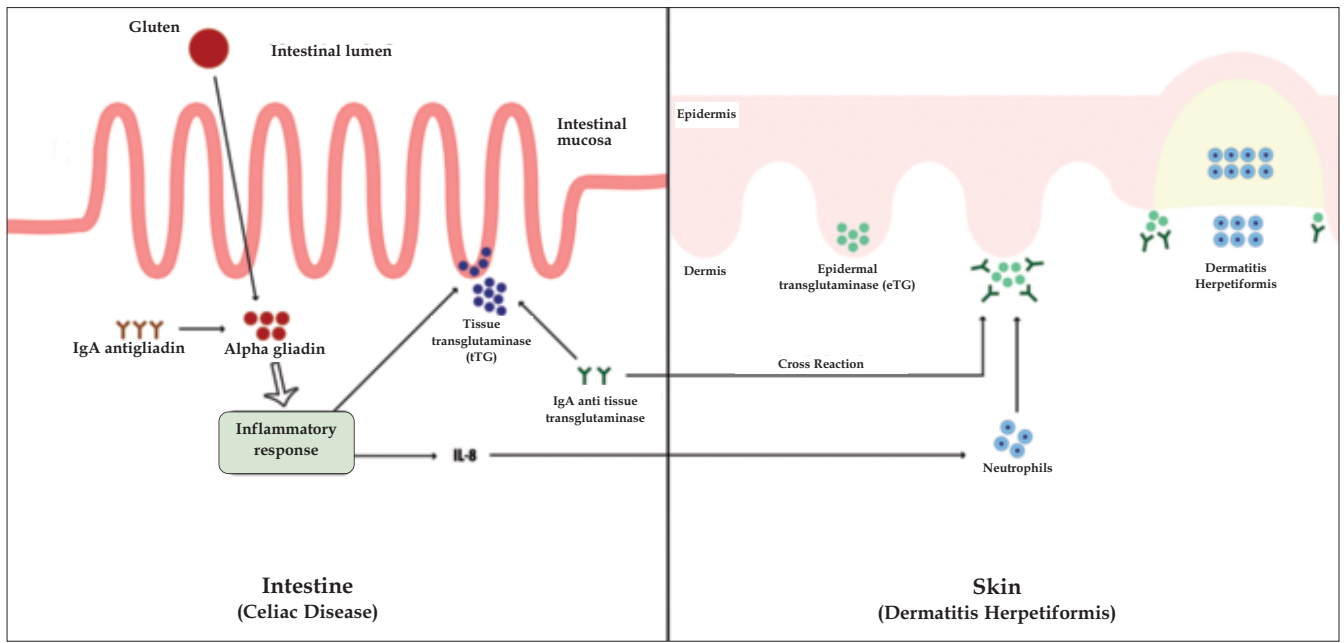

FIGURE 1:

Dermatitis Herpetiformis physiopathogenesis
Marietta et al demonstrated that IgA anti-TTG and IgA anti-ETG serum levels are dependent of intestinal inflammatory activity, suggesting that the bowel might be the site of formation of these autoantibodies. This same study did not correlate their serum levels with the degree of intestinal atrophy. ${ }^{24}$

Secretory IgA consists of two IgA chains linked by a J chain, with the latter usually being formed by the attachment of two IgA subclasses, called IgA1 and $\operatorname{IgA2}$. Serum IgA is mainly composed of IgA1. IgA deposited on the skin of individuals with DH is predominantly composed of IgA1, with minimal or undetectable IgA2 deposits. While this might suggest that this cutaneous IgA was not originated in the intestinal mucosa, it has been documented that IgA1 is the predominant immunoglobulin in gastrointestinal secretions of patients with $\mathrm{DH} .{ }^{16,30,31}$

It was also observed that the TTG could lead to gluten peptide deamidation. These peptides, compared to undeamidated ones, can induce local T lymphocyte activation, initiating the hypersensitive response to gluten. ${ }^{6,17}$

\section{b) The role of interleukin-8:}

With the occurrence of intestinal intolerance to gluten, usually in genetically predisposed individuals, the activation of CD4+ T cells located in the mucosa is triggered. Consequently, B-lymphocytes become stimulated and, in turn, start to secrete antibodies directed against $\alpha$-gliadins and autoantigens. These antibodies are predominantly of the IgA class, however some other classes of antibodies may also be produced. ${ }^{3,16}$

An inflammatory infiltrate, composed of neutrophils, can be found in skin lesions of DH patients, usually in the papillary dermis, the same place where IgA deposits occur. ${ }^{3}$ The importance of IL- 8 as one of the initiators of the neutrophilic influx has been recent- ly identified and patients with $\mathrm{DH}$ have increased serum levels of this substance. The intestinal activity, triggered by gluten in this disease, is primarily responsible for the IL- 8 production, whereas patients that are free of cutaneous lesions after the use of dapsone, but do not adhere to the diet, maintain high levels of it. Thus, we conclude that the gluten-free diet also leads to the reduction on the levels of this interleukin. ${ }^{32}$

Certain DH triggers, such as UVB exposure and trauma, have the ability to induce IL- 8 production, and thus induce the appearance of cutaneous lesions. ${ }^{33,34}$ This interleukin is a potent neutrophil activator, and acts by increasing CD11b expression and decreasing L-selectin expression by these cells, which favors their diapedesis. ${ }^{5,23,32}$ IL-8 is expressed in tissues with neutrophil infiltration and its injection into the skin results in the accumulation of neutrophils. ${ }^{35-37}$

However, despite the importance of IL-8, its elevation alone is not able to explain all of $\mathrm{DH}$ pathogenesis. Studies with transgenic mice, which expressed IL-8 in the skin, found cutaneous neutrophil infiltrates without evidence of pathological alterations in that topography. ${ }^{36}$ Similarly, transgenic mice expressing IL8 in the intestine had a local increase in neutrophils, without evidence of damage to the intestinal mucosa. ${ }^{38}$ These studies suggest that additional local factors are necessary to trigger the production of proteolytic enzymes by neutrophils. ${ }^{39}$

Besides IL-8, granulocyte-macrophage colonystimulating factor (GM-CSF) also starts being produced, especially by the dendritic cells of dermoepidermal junction. It is able to induce the expression of IgA receptors by neutrophils, which are located at the sites where $\operatorname{IgA}$ is already deposited. ${ }^{7}$ Currently, this point is deemed the probable key to better understanding the pathophysiology of this condition. ${ }^{16}$ However, the reason for the appearance of clinical lesions in specific areas such as elbows, knees and but- 
tocks is still not fully clarified, since IgA deposits also occur in places where there are no lesions, which emphasizes the influence of local factors. ${ }^{7}$

\section{enzymes:}

c) Neutrophilic infiltrate and proteolytic

The neutrophilic infiltrate in the papillary dermis is histopathologically translated as Piérard microabscesses and IgA deposits determine their location. The formation of vesicobullous lesions is derived from collagenase and elastase production by neutrophils, which leads to basement membrane destruction. ${ }^{40}$ Local keratinocytes and macrophages also start producing important enzymes, such as metalloproteinase collagenase and stromelysin- 1 which destruct the extracellular matrix. The latter seems to contribute to the formation of bullous lesions when degrading the basement membrane components such as collagen types IV and VII, and laminin-1. The process leading to the formation of these lesions usually occurs over a 24-hour period.

Studies by Airola et al sought to identify the role of these basement membrane-degrading enzymes in self-induced lesions, using $50 \%$ potassium iodine in patients with DH. Within 12 hours, even when clinical lesions were not yet visible, it was possible to identify the production of plasminogen activator urokinase by basal keratinocytes. Within 24 hours, the presence of collagenase and stromelysin- 1 in these keratinocytes was identified, these enzymes having been produced under the stimulation of plasminogen activator urokinase. At that time, it was possible to verify the destruction of laminin-1 and type VII collagen, essential components for the basement membrane integrity. Besides, laminin-5, a component of anchorage fibrils, responsible for the adhesion of keratinocytes to the basement lamina, was found in keratinocytes at the same period, possibly reflecting the local regenerative response. ${ }^{41,42}$

\section{CLINICAL PRESENTATION:}

Lesions appear as grouped vesicles on an erythematous or erythematopapulous base, predomi- nantly on the elbows, knees, and buttocks. More severe cases may present generalized involvement. ${ }^{26}$

Lesions tend to grow in a centrifugal pattern, with vesicles predominating in the periphery (Figures $2 \mathrm{~A}$ and $2 \mathrm{~B}$ ). The intense pruritus is not related to the extent of disease and promotes the loss of the herpetiform aspect due to multiple abrasions. The disease tends to evolve with periods of remissions, and there is not an efficient method to measure its activity. For this purpose, some studies used the number of lesions presented by the patient and the dose of suppressive medication administered at the time. ${ }^{3,15}$

Petechial or ecchymotic lesions may occur in the palmoplantar regions and are observed more frequently in children. Karpati studied 47 children and showed that 30 of them (64\%) had palmar purpuric lesions of a reddish-brown coloration. ${ }^{26}$

DH rarely affects the oral cavity, although IgA deposits frequently occur in this region. Studies demonstrate the difficulty of inducing the appearance of oral lesions with $50 \%$ potassium iodine. ${ }^{43}$ This involvement, when present, usually occurs especially in areas subject to trauma. Similar patterns of enamel defects in color, roughness, horizontal grooves and punctiform depressions are described in $\mathrm{DH}$ and also in $\mathrm{CD}{ }^{3}$

Atypical presentations such as palmoplantar hyperkeratosis, chronic urticaria and prurigo forms are also described. ${ }^{26}$

\section{INTESTINAL INVOLVEMENT:}

All patients with DH have intestinal sensitivity to gluten, but only a small proportion of them will present symptoms suggestive of CD. Between 20-30\% will present some degree of steatorrhea and less than $10 \%$ will have symptoms similar to $\mathrm{CD}$ such as diarrhea, cramps, and malabsorption. Reunala et al demonstrated that $16 \%$ of children with $\mathrm{DH}$ had chronic diarrhea and 10\% had iron deficiency anemia. One patient in the study had growth deficiency. ${ }^{11,44,45}$

In two thirds of patients with DH there is some degree of villous atrophy in the intestinal mucosa (Figure 3) and one third will have an increased
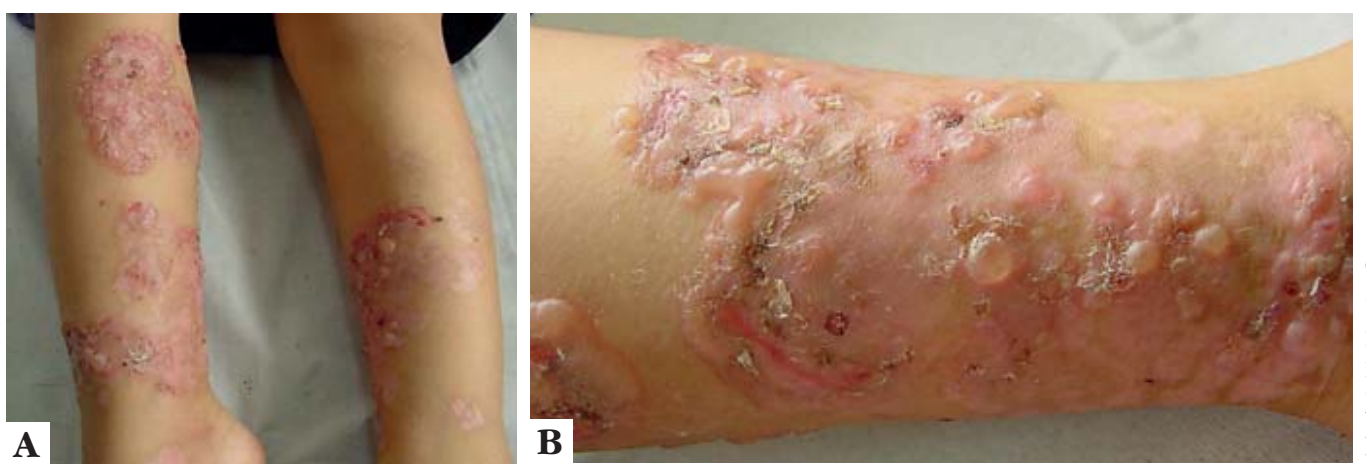

Figure 2: cutaneous lesions in a patient with $\mathrm{DH}$ : A. Vesico-bullous lesions in lower limbs; B. Grouped vesicles on an erythematous base 

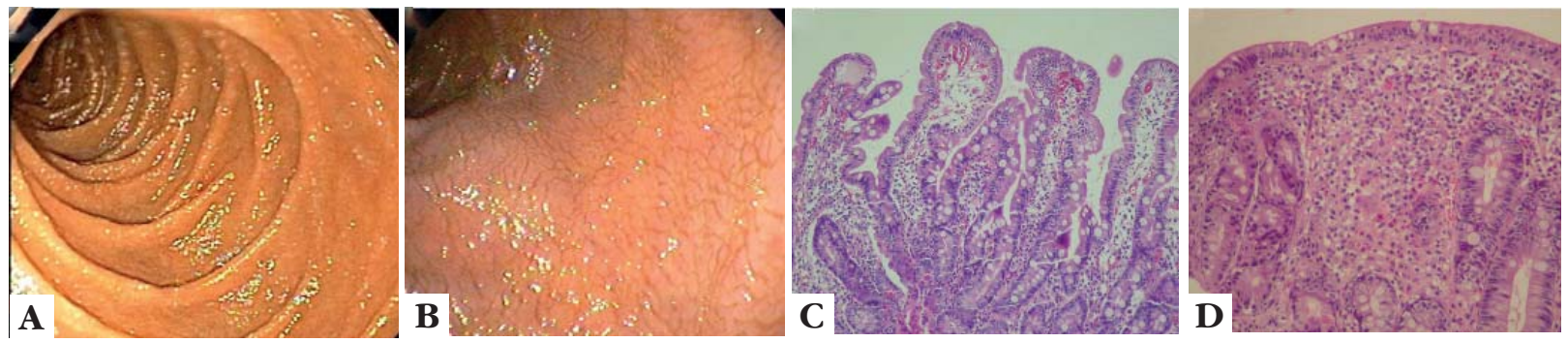

FIGURE 3: Intestinal involvement due to gluten intolerance. Colonoscopy in a healthy individual (A), and in a patient with CD (B) highlight of intestinal villous atrophy. Intestinal histopathology on a healthy individual (C), and in a patient with CD (D) note the lymphocytic infiltrate and atrophy. The images are a courtesy from Dr. Carlos Floriano de Moraes

intraepithelial lymphocyte count. ${ }^{6,30}$ Fry describes that the finding of up to 200 lymphocytes per 1,000 epithelial cells could exclude the diagnosis of DH..$^{27,46}$ Both alterations improve noticeably early with adherence to a gluten-free diet. ${ }^{15}$

\section{CORRELATED DISEASES}

Reunala et al followed 305 patients with $\mathrm{DH}$ for 10 years. The study revealed a $10 \%$ incidence of autoimmune diseases in patients with this cutaneous condition, particularly autoimmune thyroid diseases, pernicious anemia, gastric atrophy, type I diabetes, systemic lupus erythematosus, Sjoegren disease, sarcoidosis, vitiligo and alopecia areata. ${ }^{3,47}$

Cunningham and Zone evaluated 50 individuals with $\mathrm{DH}$ and showed that these patients had a high incidence of hyperthyroidism, hypothyroidism, thyroid nodules / cancer and positivity for thyroid autoantibodies. ${ }^{10,48,49}$ Gaspari et al demonstrated that the association between Hashimoto's disease and DH was not only due to HLA B8/DR3, common to both diseases, but also to the formation of thyroid autoantibodies triggered by intestinal inflammatory activity in these patients. ${ }^{49}$

Recent studies have shown an association between DH and chronic gastric atrophy. Gastric atrophy may have an autoimmune origin, predominating in the gastric body, or secondary to infection by Helicobacter pylori, usually in the gastric antrum. Alakoski et al conducted a study with 93 patients with $\mathrm{DH}$ and concluded that there was a higher incidence of gastric corpus atrophy and intestinal metaplasia when compared to controls..$^{50}$ Primignani et al studied 57 cases and found a 30\% prevalence of gastric atrophy in patients with $\mathrm{DH}$ and $15 \%$ in the control group. ${ }^{51}$ Other investigators reported even higher rates of gastric atrophy, in $50-70 \%$ of patients with $\mathrm{DH}$, and gastric hypochlorhydria in up to $90 \% .{ }^{52-56}$

\section{ASSOCIATION WITH LYMPHOMA}

Patients with DH / CD are at higher risk of developing lymphoma, ranging from 5.4 to 100 times higher than the general population..$^{57-59}$ A study conducted jointly by England and Finland demonstrated the protective effect of a gluten-free diet regarding this complication. In this study, all cases of lymphoma were related to not following the diet or having started it less than 5 years before. The occurrence of lymphoma, especially in the gastrointestinal tract and associated lymph nodes, could be a result of longterm local lymphocyte stimulation, secondary to gluten exposure. ${ }^{7}$ The majority of cases are of nonHodgkin's lymphomas, but B-cell lymphomas and Hodgkin's Disease are also reported. Some studies even found a predominance of B-cell lymphomas. ${ }^{2,60}$

Lewis et al evaluated 846 patients with $\mathrm{DH}$, and did not identify an increased risk of malignancies including lymphoma, fractures neither increased overall mortality risk among affected patients. ${ }^{61}$

\section{DIAGNOSIS}

$\mathrm{DH}$ requires important dietary changes and in most cases the use of medications with potential toxicity.

Direct immunofluorescence performed on healthy skin remains the gold standard for diagnosis. In case of a negative result, one should collect new materi$\mathrm{al}$, and determine whether the patient is on a gluten-free diet, which could lead to false-negative results. ${ }^{4}$

In patients with clinical signs that are suggestive of $\mathrm{DH}$ but with negative direct immunofluorescence, other confirmatory tests such as the dosage of anti-TTG can be used. Another possibility is the confirmation of $C D$, which warrants the initiation of specific therapy for both diseases. If there is any doubt, some authors also suggest that gluten should be vigorously offered to the patient, which would lead to the appearance of vesicular lesions over a period of 24 hours, supporting the diagnosis. ${ }^{4}$

Considering the high incidence of autoimmune diseases and associated conditions, these patients should undergo a series of tests. Certain antibodies, such as anti-thyroperoxidase antibodies or anti-TPO (positive in $20 \%$ of patients), anti gastric parietal cells (positive in $10-25 \%$ of patients), ANA, anti-La and 
anti-Ro should be measured for all CD or DH patients. Additional tests such as TSH, T3, T4 and fasting glucose levels should also be requested. ${ }^{4}$

\section{Histopathological diagnosis:}

A skin fragment for histopathological analysis must be obtained from an erythematous or erythematopapulous area near the vesicle, where neutrophilic microabscesses may be identified. Biopsy performed in vesicular lesions will show subepidermal blisters, making it difficult to differentiate from other bullous diseases with the same cleavage plane. ${ }^{3}$

The collection of neutrophils and also some eosinophils begins in the early stages, before the appearance of vesicles, especially at the top of the dermal papillae. These are Piérard microabscesses, which although very characteristic of this dermatosis, are not pathognomonic. It is also possible, at this stage, to observe the appearance of perivascular inflammatory infiltrates, especially in the superficial and middle areas of the dermis., ${ }^{3,62,63}$

Within 36-48 hours, an increase in the number of eosinophils in the infiltrate is seen in the lesions. Fragments of neutrophils can also be observed. Occasionally, neutrophilic collections may be seen in the epidermis, requiring differentiation from $\operatorname{Ig} \mathrm{A}$ pemphigus, a very rare condition. ${ }^{62,63}$

Edema with the formation of fissures or microvesicles on top of the dermal papillae occurs later. Collagen is degraded resulting in detachment of the epidermis. Multiple vesicles may coalesce forming unilocular blisters, clinically translated as vesicobullae in typical locations (Figure 4A). ${ }^{1,3}$

Warren and Cockerell reported that $37.5 \%$ of patients with $\mathrm{DH}$ have only lymphocytic infiltrate, papillary dermis fibrosis and vascular ectasia, which may be due to old and very excoriated lesions. ${ }^{26}$

\section{Immunofluorescence:}

Because of the possibility of numerous differential diagnoses, it is always desirable to perform direct immunofluorescence. For this purpose, a biopsy should be obtained from perilesional, apparently unaffected skin (up to $1 \mathrm{~cm}$ away from the lesion), where proteolytic enzymes did not cause immunoglobulin degradation. ${ }^{6}$ The specimen should be kept frozen, avoiding the denaturation of these proteins. The occurrence of $\operatorname{IgA}$ deposits along the dermal-epidermal junction and on top of the dermal papillae are the fundamental characteristics of $\mathrm{DH}$ (Figure 4B). ${ }^{1,3,6}$

IgA deposits in the dermal papillae may appear with different patterns: granular on the papillary dermis, granular on the basement membrane and fibrillar. ${ }^{26}$ Both granular patterns are often associated, so that granular deposits on the basement membrane with accentuation in the dermal papillae may be noted. Kawana and Segawa first described the fibrillar pattern in 1993, with this pattern being found in 50\% of patients in Japan. One study with 22 Chinese patients showed that $95.5 \%$ had IgA deposits with granular pattern and $4.5 \%$ with fibrillar pattern. ${ }^{23}$ Although the association between immunofluorescence fibrillar pattern with $\mathrm{DH}$ is still debated, it is believed that this pattern is related to some atypical findings, such as lack of HLA-B8/DR3DQ2, absence of circulating autoantibodies and peculiar clinical features like urticariform and psoriasiform lesions. ${ }^{23,26}$

Deposits of complement fraction 3 (C3) in dermal papillae may be present in about $50 \%$ of patients. ${ }^{3}$ Donald et al showed that after two weeks of ingestion of a gluten-containing diet, patients in a previously gluten-free diet showed an increase in $\mathrm{C} 3$ deposits on the dermal papillae. ${ }^{15}$

\section{Laboratory diagnosis:}

Investigation of anti-TTG and anti-TGE antibodies, antiendomysial antibody and more recently, deamidated anti-gliadin showed good diagnostic, although not always available.

Anti-TTG is elevated in patients with intestinal disease activity and it decreases with the adoption of

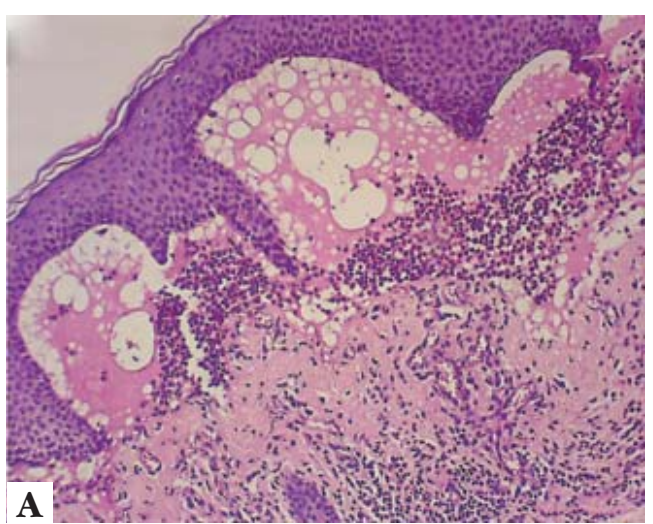

A

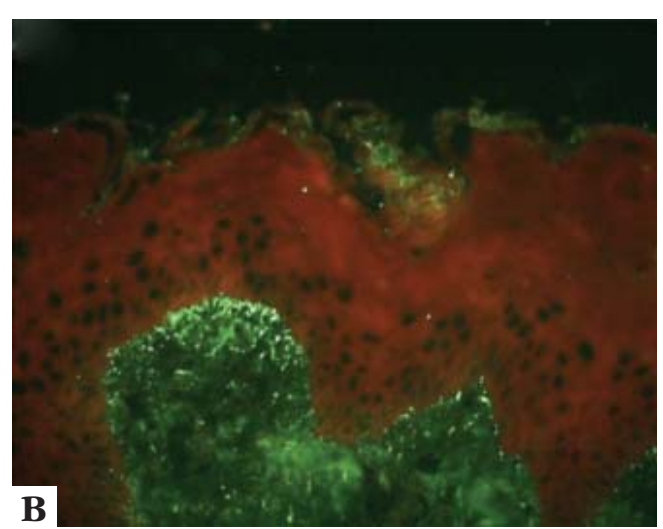

FiguRE 4:

Diagnostic methods in DH: A. Skin histopathology showing subepidermal blister with neutrophilic infiltrate in the papillary dermis; B. Direct immunofluorescence of skin fragment showing IgA deposits along the dermoepidermal junction and on top of the dermal papillae. The image is a courtesy from Dr. Carlos Floriano de Moraes; B The image is a courtesy from Dr. Valeria Aoki 
a gluten-free diet, thus being useful to assess diet adherence. ${ }^{5,26,64}$ In general, after two years of dieting, this serological marker disappears. ${ }^{6}$ The sensitivity of this antibody for cutaneous disease was $47-95 \%$ with specificity over $90 \%{ }^{4,26,65-69}$

Anti-ETG antibody has not yet been approved as a diagnostic tool in the United States. Its sensitivity is $52 \%$ in patients with $\mathrm{DH}$, but it may have lower values in the pediatric population. ${ }^{16,70,71}$ Nevertheless, other studies show up to $90 \%$ sensitivity. ${ }^{72,73}$ This divergence may be caused by the different cases severities in each study. ${ }^{16}$ This autoantibody may be present in patients with negative anti-TTG. Jaskowski et al showed that $20 \%$ of patients with $\mathrm{DH}$ have negative anti-TTG and positive anti-ETG. Accordingly, it could be used for diagnostic screening and even diet monitoring in patients with suspected $\mathrm{DH} .^{71}$

Recently some researchers have suggested the investigation of deamidated anti-gliadin antibody as a possible antigenic marker. This is particularly useful in patients with negative anti-TTG, making it the most reliable marker of gluten susceptibility. ${ }^{74-76}$ Its sensitivity ranges between 84 and $90 \% .16,77$

The analysis of anti-endomysial antibody in monkey's esophagus has also been useful, with 52$100 \%$ sensitivity for the diagnosis of $\mathrm{DH}$ and nearly $100 \%$ specificity. Similarly to anti-TTG, this marker also becomes absent in patients not exposed to gluten, and it is also used as an indicator of dietary adherence., ${ }^{4,26}$

Other antibodies can also be investigated, such as anti-gliadin antibody (conventional) and antireticulin, but both have lower accuracy compared to the aforesaid. ${ }^{26}$ Anti-gliadin and antireticulin antibodies tend to have higher positivity in CD patients compared to those with $\mathrm{DH}$, and this may reflect the lower intestinal inflammatory activity in the cutaneous form of the disease. ${ }^{6,78}$

\section{TREATMENT}

The mainstays of DH treatment are the gluten-free diet and drug therapy with sulfonamides or dapsone ${ }^{3,6,79}$

\section{Gluten-free diet:}

In 1981 Van der Meer et al reported the benefit of a gluten-free diet for patients with DH. Two patients who had improvement of their cutaneous lesions after the institution of a gluten-free diet were evaluated. Later, the patients were able to reduce the maintenance dose of dapsone. ${ }^{15,80}$ This same dietary benefit could be seen in another study by the same authors in 1985, when they recorded the resolution of cutaneous lesions in five patients, with the same dermatosis, who followed the diet. ${ }^{15,81}$

A gluten-free diet is considered the treatment of choice for patients with $\mathrm{CD} / \mathrm{DH}$, since cutaneous and intestinal manifestations are gluten-dependent, and particularly in $\mathrm{DH}$, because of the cumulative deleterious side effects of the drugs of choice. ${ }^{3,4}$ The diet usually provides gastrointestinal benefits much earlier than cutaneous ones; $\mathrm{DH}$ improvement can take up to two years to occur if only this therapy is instituted. IgA deposits may take several years to be completely eliminated from the dermoepidermal junction, however the reintroduction of gluten can lead to new deposits and rapid clinical worsening. ${ }^{4}$ The offer of gluten is also able to cause C3 deposits. ${ }^{15}$

Among other benefits of this diet, we can mention the protective effect against the development of lymphomas of the gastrointestinal tract, a benefit which is usually seen after 5 years of dieting, and also the recovery of the intestinal mucosa with consequent improvement of malabsorption in those patients with this symptom. ${ }^{3,4,644}$ More long-term studies are needed to evaluate if the diet decreases the incidence of associated auto-immune diseases. ${ }^{4}$

After a variable period of time, most patients receiving drug treatment often experience a gradual reduction and even discontinuation of medication. The mean time to dapsone interruption is 25 months. Despite the importance of following the diet, patient compliance can be quite difficult, thus nutritional monitoring and, if possible, participation in support groups are reccomended. ${ }^{3}$ Patients with $\mathrm{CD} / \mathrm{DH}$ should be instructed to carefully read food labels and avoid unknown ingredients, since many of these may be gluten derivatives. ${ }^{4}$

Cereal species that are toxic to patients with CD and $\mathrm{DH}$ belong to the Triticeae family, among which are wheat, barley and rye. Oat belongs to another family, called Avenae. Proteins that are toxic to $\mathrm{DH}$ patients are rich in proline and glutamine, known as prolamins. Oat-derived prolamin is called oat avenin, and it has a lower proline content compared to wheat, barley and rye. The content of oat avenin is $5-15 \%$, with gliadin content in wheat (wheat prolamin) being approximately $40 \%$. One study showed that 2.5 grams of avenin (equivalent to 300 grams of oat) consumed by $\mathrm{DH}$ patients who were clinically controlled with dieting, produced no deleterious effect on the skin or intestine, and neither formation of anti-endomysial, antireticulin or anti-gliadin antibodies., ${ }^{4,6}$ However, oats may be contaminated by wheat during the milling process or by the rotation technique used in agriculture crops. Thus, the ingestion of oats can be recommended to patients, provided they can ensure the sources purity. ${ }^{3,4,6,7}$

Kadunce demonstrated some degree of clinical improvement with the use of elemental diets, even in the presence of gluten, putting the latter's role in perspective. ${ }^{15}$ 


\section{Pharmacological treatment:}

Dapsone is the drug of choice in the treatment of $\mathrm{DH}$, with sulfapyridine as the alternative therapy. The mechanism of action of dapsone is not fully elucidated, but it seems to block chemotaxis and activate neutrophils besides reducing the release of leukotrienes and prostaglandins. Its administration provides fast relief from itching, and usually regression of cutaneous symptoms occurs in a week. It promotes improvement in skin lesions although it has no effect on intestinal disease. ${ }^{3,6,79}$

The commonly used dose is $100 \mathrm{mg}$ per day, with an average maintenance dose of $1 \mathrm{mg} / \mathrm{kg} /$ day. In children it is necessary to use smaller doses. ${ }^{79}$ The goal is to keep the patient under clinical control for 12 years until the benefit of diet is achieved. The lowest dose able to maintain the patient in remission should be sought and, when possible, the drug should be discontinued. Suspension of dapsone in the absence of diet will lead to recurrence of the lesions., ${ }^{3,4,6}$

The most common side effects with the use of dapsone are acute hemolytic anemia, and methemoglobinemia, which are dose-dependent. Elderly patients, especially those with comorbidities are at risk for instability of cardiac conditions, such as heart failure or coronary artery disease as a result of the decrease in hemoglobin levels. The initial reduction of hemoglobin $(2-3 \mathrm{~g})$ is common, but it is usually compensated by reticulocytosis. ${ }^{3,82}$

Deficiency of glucose-6-phosphate dehydrogenase (G6PD), especially in African-Americans and Caucasians from southern Mediterranean origin, is an absolute contraindication for the use of dapsone, because of the high-risk of severe hemolysis; although patients without G6PD deficiency may also develop this complication. ${ }^{83}$ Levels of hemoglobin, hematocrit and reticulocyte count should be monitored every two weeks during the first 3 months and after this period, every 3 months. ${ }^{3,6}$ Daily administration of 800 units of vitamin A for 4 weeks could help prevent this side effect., ${ }^{3,84}$

Methemoglobinemia is more frequent and generally more insidious than hemolysis. The occurrence of headache and lethargy after the use of dapsone may evoke suspicion. Agranulocytosis is a rare side effect, of early occurrence, appearing usually during the first 3 months of therapy. ${ }^{3}$

Dapsone can still induce severe hypersensitivity reaction with fever, rash, lymphadenopathy, and systemic involvement of varying degrees. It occurs in up to $5 \%$ of patients after 2-6 weeks of introducing the drug.,

Sulfonamides can also be used to control the acute phase of the disease, and sulfapyridine and sulfamethoxypyridazine are the most prescribed ones. The recommended dose ranges from $500 \mathrm{mg}$ to $4.5 \mathrm{~g}$ daily. Both drugs have similar side effects: hypersensi- tivity reactions, hemolytic anemia, proteinuria and crystalluria. Monitoring should be performed with $\mathrm{CBC}$ and urinalysis before the start of treatment, and monthly for the first 3 months of therapy, then twice a year after this period. ${ }^{3,49}$

Patients with rash that is hard to control even during drug therapy should limit their iodine intake because of its effect on disease exacerbation. ${ }^{3}$

\section{FOLLOW-UP}

Follow-up is required to confirm diagnosis through response to diet and also to detect and manage complications. Medical staff should evaluate these patients every 6 months to 1 year with clinical examination and consultation with the nutritionist. The following should always be monitored: diet adherence, development of related autoimmune diseases, metabolic disorders (dyslipidemia and diabetes, for example), and possible evolution to lymphoma. To monitor diet adherence, serological tests such as IgA antigliadin (especially in the deamidated form), antiendomysial and anti-transglutaminase should be ordered. However, small deviations from the diet may not be detectable., ${ }^{4,7}$

Some autoimmune cutaneous diseases such as bullous pemphigoid and pemphigus vulgaris may present clinical remission after a certain period, allowing drug therapy discontinuation. Nevertheless, longterm prognosis for $\mathrm{DH}$ remains uncertain.

Between 10 and $15 \%$ of the patients with clinical remission may interrupt the pharmacological treatment and diet. ${ }^{85-87}$ So Yeon Pack et al noted clinical remission in $12 \%$ of patients and $6 \%$ remained without lesions, but they still needed to diet. In patients with early onset of symptoms there was a smaller likelihood of remission. ${ }^{88}$

Although patients with $\mathrm{DH} / \mathrm{CD}$ have increased risk of developing lymphoma and autoimmune diseases, the mortality rate was not higher amongst them in studies. A slight increase in survival was evidenced in patients adhering to a gluten-free diet, especially due to reduction of atherosclerotic disorders such as coronary artery disease. This is the result of lower levels of cholesterol and triglycerides seen in these patients. ${ }^{7,89,90}$

\section{FINAL CONSIDERATIONS}

Researches on $\mathrm{DH}$, especially about the elucidation of its pathogenesis, have shown that this is not simply a bullous cutaneous disease, but a cutaneous-intestinal disorder cause by gluten hypersensitivity. Its association with $\mathrm{CD}$ is well established, with both diseases being transmitted by HLA-DQ2 and HLA-DQ8 alleles.

Exposure to gluten remains as the starting point that triggers a not yet fully elucidated inflammatory 
cascade. Alpha gliadins, especially their N-terminal portion, have high immunogenic potential in these individuals. And this inflammatory process starts the formation of autoantibodies, the key to understanding the cutaneous involvement.

IgA begins to recognize its own tissue transglutaminase and these autoantibodies, through crossreaction, begin the attack on transglutaminase in the epidermis, where neutrophil collections will later be found. These in turn produce a number of extracellular matrix-degrading enzymes culminating in the appearance of vesicobullous lesions.

Lesions tend to be very pruriginous and are most often located on the elbows, knees and buttocks. A number of autoimmune diseases may be associated and the minimum required investigation must be performed. Progression to lymphoma can be seen in several studies, although with a widely varying frequen$c y$, it tends to occur mainly in the gastrointestinal tract of individuals who do not comply with the diet.

Direct immunofluorescence remains the gold standard test to confirm diagnosis, but various authors consider a compatible clinical presentation plus specific supporting serum testing as a confirmatory possibility. Some tests, in addition to helping diagnose the disease, can assist in the evaluation of dietary adherence.

A gluten-free diet is essential in the treatment of patients with $\mathrm{CD} / \mathrm{DH}$, since cutaneous and intestinal clinical manifestations are gluten-dependent, and improve with suspension of its intake. Benefits such as improvement of the intestinal mucosal atrophy, reduced risk of lymphoma and improved malabsorptive syndrome are usually noticed. The gluten-free diet diminishes lymphocyte infiltration in the dermal papilla, reduces IL-8 production and decreases IgA deposits. Dapsone remains the primary drug for treatment, but it requires monitoring of possible side effects, some with potential lethality.

The disease has a low rate of remission, around $10-15 \%$ even in the long term, but increase in mortality has not been detected in this group, despite the possibility of association with autoimmune diseases and lymphomas.]

\section{REFERENCES}

1. Wojnarowska F, Venning VA. Immunobullous Diseases. In: Burns T, Breathnach S, Cox N, Griffiths C, editors. Rook`s Textbook of Dermatology. Hardcover: WileyBlackwell; 2010. p. 58-62.

2. Hervonen K, Karell K, Holopainen P, Collin P, Partanen J, Reunala T. Concordance of dermatitis herpetiformis and celiac disease in monozygous twins. J Invest Dermatol. 2000;115:990-3.

3. Jacques CMC. Dermatite herpetiforme e dermatose por IgA Linear. In: Ramos-eSilva M, Castro MCR. Fundamentos de Dermatologia. Rio de Janeiro: Atheneu; 2009. p. 653-8.

4. Caproni M, Antiga E, Melani L, Fabbri P.; Italian Group for Cutaneous Immunopathology. Guidelines for the diagnosis and treatment of dermatitis herpetiformis. J Eur Acad Dermatol Venereol. 2009;23:633-8.

5. Bonciani D, Verdelli A, Bonciolini V, D'Errico A, Antiga E, Fabbri P, et al. Dermatitis herpetiformis: from the genetics to the development of skin lesions. Clin Dev Immunol. 2012;2012:239691.

6. Fry L. Dermatitis herpetiformis: problems, progress and prospects. Eur J Dermatol. 2002;12:523-31.

7. Reunala TL. Dermatitis herpetiformis. Clin Dermatol. 2001;19:728-36.

8. Fry L, Keir P, McMinn RM, Cowan JD, Hoffbrand AV. Small intestinal structure and function and haematological changes in dermatitis herpetiformis. Lancet. 1967;2:729-33.

9. Fry L, Seah PP, Riches DJ, Hoffbrand AV. Clearance of skin lesions in dermatitis herpetiformis after glúten withdrawal. Lancet. 1973;1:288-91.

10. Petersen M, Zone J. The relationship between autoimmunes bullous disease and systemic disorders. J Invest Dermatol. 2008;128:E28-30.

11. Gawkrodger DJ, Blackwell JN, Gilmour HM, Rifkind EA, Heading RC, Barnetson RS. Dermatitis herpetiformis diagnosis diet and demography. Gut. 1984;25:151-7.

12. Mobacken $H$, Kastrup W, Nilsson LA. Incidence and prevalence of dermatitis herpetiformis in Sweden. Acta Derm Venereol. 1984;64:400-4.

13. Reunala $\mathrm{T}$. Incidence of familial dermatitis herpetiformis. $\mathrm{Br} \mathrm{J}$ Dermatol. 1996;134:394-8.

14. Spurkland A, Ingvarsson G, Falk ES, Knutsen I, Sollid LM, Thorsby E. Dermatitis herpetiformis and celiac disease are both primarily associated with the HLA-DQ $\left(\alpha 1^{\star} 0501, \beta 1^{\star} 02\right)$ or the HLA-DQ $\left(\alpha 1^{\star} 03, \beta 1^{\star} 0302\right)$ heterodimers. Tissue Antigens. 1997;49:29-34.

15. Kadunce DP, McMurry MP, Avots-Avotins A, Chandler JP, Meyer LJ, Zone JJ. The effect of an Elemental diet with and without glúten on disease activity in dermatitis herpetiformis. J Invest Dermatol. 1991;97:175-82.

16. Gornowicz-Porowska J, Bowszyc-Dmochowska M, Seraszek-Jaros A, Kaczmarek E, Dmochowski M. Association between levels of IgA antibodies to tissue transglutaminase and gliadin-related nonapeptides in dermatitis herpetiformis. Scientific World Journal. 2012;2012:363296.

17. Dieterich W1, Laag E, Bruckner-Tuderman L, Reunala T, Kárpáti S, Zágoni T, et al. Antibodies to tissue transglutaminase as serologic markers in patients with dermatitis herpetiformis. J Invest Dermatol. 1999;113:133-6

18. Molberg 0, Mcadam SN, Körner R, Quarsten H, Kristiansen C, Madsen L,et al. Tissue transglutaminase selectively modifies gliadin peptides that are recognized by gut-derived T cells in celiac disease. Nat Med. 1998;4:713-7.

19. Nemes Z, Fésüs L, Egerházi A, Keszthelyi A, Degrell IM. Nع (Y-glutamyl) lysine in cerebrospinal fluid marks Alzeimer type and vascular dementia. Neurobiol Aging. 2001;22:403-6. 
20. Jeitner TM, Bogdanov MB, Matson WR, Daikhin Y, Yudkoff M, Folk JE, et al. Nع ( $\gamma-$ L-glutamyl)-L-lysine (GGEL) in increased in cerebrospinal fluido $f$ patients with Huntington's disease. J Neurochem. 2001;79:1109-12.

21. Sárdy M, Kárpáti S, Merkl B, Paulsson M, Smyth N. Epidermal transglutaminase (TGase 3) is the autoantigen of dermatitis herpetiformis. J Exp Med. 2002;195:747-57.

22. Hitomi, K. Transglutaminases in skin epidermis. Eur J Dermatol. 2005;15:313-9.

23. Nakajima K. Recent advances in dermatitis herpetiformis. Clin Dev Immunol. 2012;2012:914162

24. Marietta EV, Camilleri MJ, Castro LA, Krause PK, Pittelkow MR, Murray JA. Transglutaminase autoantibodies in dermatitis herpetiformis and celiac sprue. J Invest Dermatol. 2008;128:332-5.

25. Karpati S. Dermatitis herpetiformis: close to unraveling a disease. J Dermatol Sci. 2004:34:83-90.

26. Bonciolini V1, Bonciani D, Verdelli A, D'Errico A, Antiga E, Fabbri P, et al. Newly described clinical and immunopathological feature of dermatitis herpetiformis. Clin Dev Immunol. 2012;2012:967974..

27. Donaldson MR, Zone JJ, Schmidt LA, Taylor TB, Neuhausen SL, Hull CM,et al. Epidermal transglutaminase deposits in perilesional and uninvolved skin in patients with dermatitis herpetiformis. J Invest Dermatol. 2007:127:1268-71.

28. Helin H, Mustonen J, Reunala T, Pasternack A.IgA nephropathy associated with celiac disease and dermatitis herpetiformis. Arch Pathol Lab Med. 1983;107:324-7.

29. Reunala T, Helin H, Pasternack A, Linder E, Kalimo K. Renal involvement and circulating immune complexes in dermatitis herpetiformis. J Am Acad Dermatol. $1983 ; 9: 219-23$

30. Hall RP III. Progress in dermatology. J Invest Dermatol. 1992;99:873-81.

31. Olbricht SM, Flotte TJ, Collins AB, Chapman CM, Harrist TJ. Dermatitis herpetiformis: cutaneous deposition of polyclonal IgA1. Arch Dermatol. 1986;122:418-21.

32. Hall RP 3rd, Takeuchi F, Benbenisty KM, Streilein RD. Cutaneous endothelial cell activation in normal skin of patients with dermatitis herpetiformis associated with increased serum levels of IL-8, sE-selectin and TNF- $\alpha$. J Invest Dermatol. 2006;126:1331-7.

33. Strickland I, Rhodes LE, Flanagan BF, Friedmann PS. TNF-alpha and IL-8 are upregulated in the epidermis of normal human skin after UVB exposure: correlation with neutrohil accumulation and E-selectin expression. J Invest Dermatol. 1997;108:763-8.

34. Takeuchi F, Sterilein RD, Hall RP 3rd. Increased E-selectin, IL-8 and IL-10 gene expression in human skin after minimal trauma: a potencial explanation of regional distribuition of skin lesions. Exp Dermatol. 2003;12:777-83.

35. Douglass J, Dhami D, Bulpitt M, Lindley IJ, Shute J, Church MK, et al. Intradermal challenge with interleukin-8 causes tissue oedema and neutrophil accumulation in atopic and non-atopic human subjects. Clin Exp Allergy. 1996;26:1371-9.

36. Lira SA, Zalamea P, Heinrich JN, Fuentes ME, Carrasco D, Lewin AC, et al. Expression of the chemokine N51/KC in the thymus and epidermis of transgenic mice results in marked infiltration of a single class of inflammatory cells. J Exp Med. 1994:180:2039-48.

37. Murphy PM. Neutrophil receptors for interleukin-8 and related CXC chemokines. Semin Hematol. 1997;34:311-8.

38. Kucharzik T, Hudson JT 3rd, Lügering A, Abbas JA, Bettini M, Lake JG,et al. Acute induction of human IL-8 by intestinal epithelium triggers neutrophil infiltration without mucosal injury. Gut. 2005;54:1565-72.

39. Hall RP 3rd, Benbenisty KM, Mickle C, Takeuchi F, Streilein RD. Serum IL-8 in patients with dermatitis herpetiformis is produced in response to dietary glúten. $\mathrm{J}$ Invest Dermatol. 2007;127:2158-65.

40. Oikarinen Al, Reunala T, Zone JJ, Kiistala U, Uitto J. Proteolytic enzymes in blister fluids from patients with dermatitis herpetiformis. Br J Dermatol. 1986;114:295-302.

41. Airola K, Vaalamo M, Reunala T, Saarialho-Kere UK. Enhanced expression of interstitial collagenase, stromelysin-1, and urokinase plasminogen activator in tesions of dermatitis herpetiformis. J Invest Dermatol. 1995;105:184-9.

42. Airola K, Reunala T, Salo S, Saarialho-Kere UK.. Urokinase plasminogen activator is expressed by stromelysin-1, and laminin- 5 in experimentally induced dermatitis herpetiformis lesions. J Invest Dermatol. 1997;108:7-11.

43. Patinen P, Hietane J, Malmström M, Reunala T, Savilahti E. lodine and gliandin challenge on oral mucosa in dermatitis herpetiformis. Acta Derm Venereol. 2002;82:86-9.

44. Reunala T, Kosnai I, Karpati S, Kuitunen P, Török E, Savilahti E. Dermatitis herpetiformis: jejunal findings and skin response to gluten free diet. Arch Dis Child. 1984:59:517-22.

45. Reunala T, Blomqvist K, Tarpila S, Halme H, Kangas K. Gluten-free diet in dermatitis herpetiformis: clinical response of skin lesions in 81 patients. $\mathrm{Br} \mathrm{J}$ Dermatol. 1977:97:473-80.
46. Fry L, Seah PP, McMinn RM, Hoffbrand AV. Lymphocytic infiltration of epithelium in diagnosis of glúten-sensitive enteropathy. Br Med J. 1972;3:371-4.

47. Reunala T, Collin P. Diseases associated with dermatitis herpetiformis. $\mathrm{Br} J$ Dermatol. 1997;136:315-8.

48. Cunningham MJ, Zone JJ. Thyroid abnormalities in dermatitis herpetiformis: prevalence of clinical thyroid disease and thyroid antibodies. Ann Intern Med. 1985;102:194-6.

49. Gaspari AA, Huang CM, Davey RJ, Bondy C, Lawley TJ, Katz SI. Prevalence of thyroid abnormalities in patients with dermatitis herpetiformis and in control subjects with HLA-B8/-DR3. Am J Med. 1990;88:145-50.

50. Alakoski A, Salmi TT, Hervonen K, Kautiainen H, Salo M, Kaukinen K,et al. Chronic gastritis in dermatitis herpetiformis: a controlled study. Clin Dev Immunol. 2012;2012:640630.

51. Primignani M, Agape D, Ronchi G, Forzenigo L, Bonato C, Meroni P, et al. Gastric histology and function tests in Italian patients with dermatitis herpetiformis. Scand J Gastroenterol. 1990;25:357-62.

52. Heading RC, Parkin DM, Barnetson RS, McClelland DB, Shearman DJ. Small-intestinal bacterial flora in dermatitis herpetiformis. Am J Dig Dis. 1974;19:704-8.

53. Anderson H, Dotevall G, Mobacken H. Gastric secretion of acid and intrinsic factor in dermatitis herpetiformis. Scand J Gastroenterol. 1971;6:411-6.

54. Fausa 0, Larsen TE, Husby G, Thune P. Gastrointestinal investigations in dermatitis herpetiformis. Acta Derm Venereol. 1975;55:203-6.

55. Stockbrügger R, Andersson H, Gillberg R, Kastrup W, Lundquist G, Mobacken H Autoimmune atrophic gastrics in patients with dermatitis herpetiformis. Acta Derm Venereol. 1976;56:111-3.

56. O'Donoghue DP, Lancaster-Smith M, Johnson GD, Kumar PJ. Gastric lesion in dermatitis herpetiformis. Gut. 1976;17:185-8.

57. Collin P, Pukkala E, Reunala T. Malignancy and survival in dermatitis herpetiformis: A comparison to coeliac disease. Gut. 1996;38:528-30.

58. Leonard JN, Tucker WF, Fry JS, Coulter CA, Boylston AW, McMinn RM, et al. Increased incidence of malignancy in dermatitis herpetiformis. Br Med J (Clin Res Ed). 1983;286:16-8.

59. Sigurgeirsson B, Agnarsson BA, Lindelöf B. Risk of lymphoma in patients with dermatitis herpetiformis. BMJ. 1994;308:13-5.

60. Hervonen $\mathrm{K}$, Vornanen M, Kautiainen H, Collin P, Reunala T. Lymphoma in patients with dermatitis herpetiformis and their first-degree relatives. $\mathrm{Br} \mathrm{J}$ Dermatol. 2005;152:82-6.

61. Lewis NR, Logan RF, Hubbard RB, West J. No increase in risk of fracture, malignancy or mortality in dermatitis herpetiformis: a cohort study. Aliment Pharmacol Ther. 2008;27:1140-7

62. Weedon D. Skin Pathology. 3rd ed. London: Churchill Livingstone; 2010. p. 346-51.

63. Faure M. Dermatitis herpetiformis. Semin Dermatol. 1988;7:123-9.

64. Cunha PR, Barraviera SRCS. Autoimmune bullous dermatoses. An Bras Dermatol. 2009;84:111-24.

65. Desai AM, Krishnan RS, Hsu S. Medical Pearl: using tissue transglutaminase antibodies to diagnose dermatitis herpetiformis. J Am Acad Dermatol. 2005;53:867-8.

66. Porter WM, Unsworth DJ, Lock RJ, Hardman CM, Baker BS, Fry L. Tissue transglutaminase antibodies in dermatitis herpetiformis. Gastroenterology. 1999;117:749-50

67. Koop I, Ilchmann R, Izzi L, Adragna A, Koop H, Barthelmes H. Detection of autoantibodies against tissue transglutaminase in patients with celiac disease and dermatitis herpetiformis. Am J Gastroenterol. 2000;95:2009-14.

68. Caproni M, Cardinali C, Renzi D, Calabrò A, Fabbri P. Tissue transglutaminase antibody assessment in dermatitis herpetiformis. Br J Dermatol. 2001;144:196-7.

69. Kumar V1, Jarzabek-Chorzelska M, Sulej J, Rajadhyaksha M, Jablonska S. Tissue transglutaminase and endomysial antibodies: diagnostic markers of gluten-sensitive enteropathy in dermatitis herpetiformis. Clin Immunol. 2001;98:378-82.

70. Hull CM, Liddle M, Hansen N, Meyer LJ, Schmidt L, Taylor T, et al. Elevation of IgA anti-epidermal transglutaminase antibodies in dermatitis herpetiformis. $\mathrm{Br} \mathrm{J}$ Dermatol. 2008;159:120-4.

71. Jaskowski TD, Hamblin T, Wilson AR, Hill HR, Book LS, Meyer LJ, et al. IgA antiepidermal transglutaminase antibodies in dermatitis herpetiformis and pediatric celiac disease. J Invest Dermatol. 2009;129:2728-30.

72. Rose C, Bröcker EB, Zillikens D. Clinical, histological and immunpathological findings in 32 patients with dermatitis herpetiformis Duhring. J Dtsch Dermatol Ges. 2010;8:265-70, 265-71.

73. Rose C, Armbruster FP, Ruppert J, Igl BW, Zillikens D, Shimanovich I. Autoantibodies against epidermal transglutaminase are a sensitive diagnostic marker in patients with dermatitis herpetiformis on a normal or gluten-free diet. J Am Acad Dermatol. 2009;61:39-43.

74. Jaskowski TD, Donaldson MR, Hull CM, Wilson AR, Hill HR, Zone JJ,et al. Novel screening assay performance in pediatric celiac disease and adult dermatitis herpetiformis. J Pediatr Gastroenterol Nutr. 2010;51:19-23. 
75. Sugai E, Hwang HJ, Vázquez H, Smecuol E, Niveloni S, Mazure R,et al. New serology assays can detect gluten sensitivity among enteropathy patients seronegative for anti-tissue transglutaminase. Clin Chem. 2010;56:661-5.

76. Sugai E, Smecuol E, Niveloni S, Vázquez H, Label M, Mazure R, et al. Celiac disease serology in dermatitis herpetiformis: which is the best option for detecting gluten sensitivity? Acta Gastroenterol Latinoam. 2006;36:197-201.

77. Kasperkiewicz M, Dähnrich C, Probst C, Komorowski L, Stöcker W, Schlumberger $W$, et al. Novel assay for detecting celiac disease-associated autoantibodies in dermatitis herpetiformis using deamidated gliadin-analogous fusion peptides. J Am Acad Dermatol. 2012;66:583-8.

78. Lane AT, Huff JC, Weston W. Detection of glúten in human será by na enzime immunoassay: comparison of dermatitis herpetiformis and celiac disease patients with normal controls. J Invest Dermatol. 1982;79:186-9.

79. Tristani-Firouzi P, Zone JJ. Dermatite herpetiforme. In: Lebwohl MG, Heymann WR, Berth-Jones J, Coulson I. Tratamento de doenças de pele: estratégias terapêuticas abrangentes. Barueri: Manole; 2004. p. 151-3.

80. van der Meer JB, Zeedijk N, Poen H, van der Putte SC. Rapid improvement of dermatitis herpetiformis after elemental diet. Arch Dermatol Res. 1981;271:455-9.

81. Zeedijk N, van der Meer JB, Poen H, van der Putte SC. Dermatitis herpetiformis: consequences of elemental diet. Acta Derm Venereol. 1986;66:316-20.

82. Smith EP, Zone JJ. Dermatitis herpetiformis and linear IgA bullous dermatosis. Dermatol Clin. 1993;11:511-26.

83. Cream JJ, Scott GL. Anaemia in dermatitis herpetiformis. The role of dapsoneinduced haemolysis and malabsorption. Br J Dermatol. 1970;82:333-42.

84. Prussick R, Ali MA, Rosenthal D, Guyatt G. The protective effect of vitamin E on the hemolysis associated with dapsone treatment in patients with dermatitis herpetiformis. Arch Dermatol. 1992;128:210-3.

85. Hull C, Zone J. Dermatitis herpetiformis and linear IgA bullous dermatosis. In: Bolognia J, Jorizzo J, Rapini R, editors. Dermatology. 2nd ed. Philadelphia, PA: Mosby; 2008. p.447-52.

86. Garioch JJ, Lewis HM, Sargent SA, Leonard JN, Fry L. 25 years' experience of glúten-free diet in the treatment of dermatitis herpetiformis. $\mathrm{Br} J$ Dermatol. 1994;131:541-5.

87. Fry L. Dermatitis herpetiformis. Baillieres Clin Gastroenterol. 1995;9:371-93.

88. Paek SY, Steinberg SM, Katz SI. Remission in dermatitis herpetiformis. Arch Dermatol. 2011;147:301-5.

89. Swerdlow AJ, Whittaker S, Carpenter LM, English JS. Mortality and cancer incidence in patients with dermatitis herpetiformis. Br J Dermatol. 1993;129:140-4.

90. Lear JT, Neary RH, Jones P, Fitzgerald DA, English JS. Risk factors for ischaemic hearth disease in patients with dermatitis herpetiformis. J R Soc Med. 1997;90:247-9.

\author{
MAILING ADDRESS: \\ Marcos Vinícius Clarindo \\ Rua Universitária, 1619. \\ Jardim Universitário \\ 85819-110 - Cascavel - PR. \\ E-mail:marcosviniciusdoc@hotmail.com
}

How to cite this article: Clarindo MV, Possebon AT, Soligo EM, Uyeda H, Ruaro RT, Empinotti JC. Dermatitis Herpetiformis: pathophysiology, clinical presentation, diagnosis and treatment. An Bras Dermatol. 2014;89(6):865-77. 


\section{QUESTIONS}

1. We can find some peculiarities in Asian individuals with $\mathrm{DH}$ such as the lowest association with DC. Which of the following is another DH characteristic related to this race?

a) It occurs more often in females.

b) Dermatologic manifestations begin at older age.

c) There is a greater association with autoimmune diseases.

d) It causes IgA deposits with a fibrillar pattern.

\section{About gluten, it is incorrect to state that:}

a) It is found in foods such as wheat, barley and rye.

b) It is common that oat is contaminated with wheat due to milling processes.

c) It has two peptides, gliadin and glutenin.

d) Beta-gliadins are the most immunogenic ones.

\section{About TTG, it is incorrect to state that:}

a) It has $64 \%$ structural homology when compared to ETG.

b) It is considered the main serological marker in CD.

c) It seems to be involved in the pathogenesis of other diseases such as Alzheimer's disease.

d) It is the cutaneous target in $\mathrm{DH}$.

\section{About ETG, it is incorrect to state that:}

a) It is also known as TG3, and it is located especially in keratinocytes.

b) One of its functions is the maintenance of the stratum corneum's integrity.

c) Sera from patients with DH react preferentially against TTG.

d) Its structure, similar to TTG allows the occurrence of crossreactivity between them.

5. Regarding $\mathrm{CD}$ and $\mathrm{DH}$ pathogenesis, it is correct to state that: a) TTG can cause gluten peptide deamidation.

b) IgA anti-TTG and IgA anti-ETG serum levels do not depend on intestinal inflammatory activity.

c) IgA anti-ETG antibodies are formed in the epidermis.

d) There are no reports of other IgA-mediated diseases in patients with $\mathrm{DH}$.

6. Regarding interleukin-8, it is incorrect to state that:

a) In $\mathrm{DH}$ it is produced particularly in the epidermis

b) It is one of the causes of the characteristic neutrophilic influx in this disease.

c) Patients without cutaneous lesions, who do not follow the diet, maintain a higher production of IL-8.

d) There may be an increase in the production of IL- 8 after sun exposure and the occurrence of trauma.

7. Regarding vesicobullous lesion formation in $\mathrm{DH}$, it is incorrect to state that:

a) GM-CSF production appears to be important to attract neutrophils to areas with IgA deposits.

b) $\operatorname{IgA}$ is usually deposited only in areas that are most affected by the disease, such as elbows, knees and buttocks.

c) The formation process of these lesions usually occurs within 24 hours.

d) Within 24 hours of evolution it is possible to identify the presence of collagenase and stromelysin-1 in regional keratinocytes.

8. Regarding DH clinical presentation, it is correct to affirm that:

a) Pruritus is usually more intense in patients with more exuberant lesions. b) Areas that are more pruritic usually present more characteristic lesions.

c) Petechial and purpuric lesions usually occur on the trunk region, especially in adults.

d) Palmoplantar hyperkeratosis can be a cutaneous manifestation of $\mathrm{DH}$

9. Regarding oral cavity involvement in $\mathrm{DH}$, it is correct to say that:

a) $\mathrm{DH}$ often affects the oral cavity.

b) IgA deposits rarely occur in this region, since salivary enzymes cause their degradation.

c) This involvement, when present, usually spares areas that are subject to trauma.

d) $\mathrm{CD}$ and $\mathrm{DH}$ can present similar involvements in oral cavity.

10. Regarding DH gastrointestinal involvement, it is correct to affirm that:

a) Most patients have steatorrhea.

b) Most patients have chronic diarrhea.

c) Most patients show atrophy of intestinal villi.

d) Intraepithelial neutrophil count increase is common, even in patients without intestinal atrophy.

11. Which of the following endocrinopathies is most associated with DH?
a) Acromegaly
b) Type I diabetes
c) Type II diabetes
d) Hashimoto's disease

12. Which of the following tests is less useful in the investigation of patients with $\mathrm{DH}$ ?
a) FAN
b) Anti-gastric parietal cells antibodies
c) HBSAg
d) Anti-TPO (Anti-thyroperoxidase antibodies)

13. Regarding the association between $\mathrm{DH}$ and lymphomas, it is correct to state that:

a) Hodgkin's lymphomas predominate in these patients.

b) The occurrence of B-cell lymphoma associated with HD has not yet been reported.

c) It usually affects extraintestinal lymph nodes.

d) A gluten-free diet reduces the occurrence of this complication, especially after 5 years of adherence to it.

14. Mark the incorrect alternative regarding DH investigation:

a) The skin fragment for histopathological analysis must be extracted from an erythematous or erythematopapulous area adjacent to the vesicles.

b) Piérard microabscesses are neutrophilic collections that appear especially in the reticular dermis.

c) Neutrophilic infiltration can reach the epidermis, in which case it must be differentiated from IgA pemphigus.

d) Old or heavily excoriated lesions may not present the typical findings during histopathological examination.

15. Regarding DIF in the investigation of $\mathrm{DH}$, it is incorrect to state that:

a) DIF remains the gold standard for the diagnosis of $\mathrm{DH}$.

b) DIF should be performed preferentially in a vesicular lesion, since this region is rich in IgA deposits. 
c) IgA deposits with fibrillar patterns seem to occur preferentially in Asian patients.

d) Deposits of complement fraction 3 in dermal papillae may occur in about $50 \%$ of patients.

16. Which of the following exams has the lowest accuracy for DH diagnosis?
a) Anti TTG
b) Anti ETG
c) Deamidated anti-gliadin
d) Antireticulin

17. Which of the following benefits could not yet be verified in patients with $\mathrm{DH}$ on a gluten-free diet?
a) Better control of cutaneous symptoms.
b) Better control of intestinal symptoms.
c) Lower doses of dapsone required to control cutaneous symptoms.
d) Lower association with autoimmune diseases.

18. Regarding the use of dapsone in the treatment of $\mathrm{DH}$, it is incorrect to state that:
a) It promotes rapid improvement in pruritus and cutaneous lesions.
b) Lower doses for clinical control may be needed if the patient is on a gluten-free diet.
c) Hemolytic anemia and methemoglobinemia, likely side effects, are dose-dependent.
e) Agranulocytosis is a possible late complication of this drug.

19. What percentage of patients with $\mathrm{DH}$ reaches clinical remission of the disease and may be without dieting and without pharmacological treatment?
a) $0-5 \%$
b) $10-15 \%$
c) $20-25 \%$
d) $30-35 \%$

20. Some studies demonstrate that patients with DH may have a higher survival rate than the normal population. Which of the following expresses the most likely cause of this finding?
a) Lower rates of atherosclerotic disease.
b) Lower BP values.
c) Lower glycemic index.
d) Lower risk of cancer.

\section{Answer key}

Complementary exams in the diagnosis of american tegumentary leishmaniasis. An Bras Dermatol. 2014;89(5):701-711.

$\begin{array}{llll}\text { 1) } \mathrm{B} & \text { 6) } \mathrm{D} & \text { 11) } \mathrm{A} & \text { 16) } \mathrm{C} \\ \text { 2) } \mathrm{B} & \text { 7) } \mathrm{A} & \text { 12) } \mathrm{D} & \text { 17) } \mathrm{B} \\ \text { 3) } \mathrm{D} & \text { 8) } \mathrm{C} & \text { 13) } \mathrm{C} & \text { 18) } \mathrm{A} \\ \text { 4) } \mathrm{A} & \text { 9) } \mathrm{B} & \text { 14) } \mathrm{B} & \text { 19) } \mathrm{C} \\ \text { 5) } \mathrm{B} & \text { 10) } \mathrm{C} & \text { 15) } \mathrm{C} & \text { 20) } \mathrm{D}\end{array}$

\section{Papers}

Information for all members: The EMC-D questionnaire is now available at the homepage of the Brazilian Annals of Dermatology: www.anaisdedermatologia.org.br. The deadline for completing the questionnaire is 30 days from the date of online publication. 